\title{
Polar Lows Over the Eastern Part of the Eurasian Arctic: The Sea-Ice Retreat Consequence
}

\author{
Zabolotskikh Elizaveta V. ${ }^{1}$, Gurvich Irina A. ${ }^{2}$, Chapron Bertrand ${ }^{3}$ \\ ${ }^{1}$ Russian State Hydrometeorol Univ, Satellite Oceanog Lab, St Petersburg 195196, Russia. \\ ${ }^{2}$ Russian Acad Sci, VI llichev Pacific Oceanol Inst, Far Eastern Branch, POI FEB RAS, Vladivostok \\ 690041, Russia. \\ ${ }^{3}$ Inst Francais Rech Exploitat Mer, F-29280 Plouzane, France.
}

Corresponding author email address : liza@rshu.ru; gurvich@poi.dvo.ru; nreul@ifremer.fr

\begin{abstract}
:
With the sea-ice decline over the eastern part of the Eurasian Arctic (EEA), polar mesocyclones (MCs) and their most intensive representatives-polar lows (PLs)-can occur over more open-water areas. Visible and infrared MODIS images, active and passive microwave spaceborne instruments, and ERA Interim reanalysis data are combined and used to analyze the synoptic situations and to infer the factors influencingMC appearance and evolution over the Kara Sea, the Laptev Sea, the East Siberian Sea, and the Chukchi Sea. In recent years, the Arctic more often loses its summer sea-ice cover, and PLs may more commonly emerge within open-water Eastern Arctic regions during fall and summer months. This conclusion is derived basing on the analysis of more than 150 MCs for August, September, October, and November months over the EEA for the period of 2003-2015. PL and MC distribution and characteristics necessitate to be studied with a multisensor approach since neither reanalysis data nor data from a single instrument can provide sufficient means for their confident detection and analysis.
\end{abstract}

Keywords : Arctic, geoscience, mesoscale cyclones

1 Introduction

For a long time, the Arctic Ocean has been considered as a "serene region" with the sea ice layer acting to stabilize the near surface interactions. This situation is now changing and summer sea-ice extents are often reported to vanish [1]. The rapid loss of the summer sea-ice cover can affect the way that the sea ice, ocean and atmosphere interact at different levels. In particular, sea ice decline, especially over the Eastern part of the Eurasian Arctic, can now trigger more often favorable conditions to foster the formation of mesocyclones (MCs), including the most intensive - polar lows (PLs). Various sea ice decline effects, both observed and modeled, are discussed in [2]. The general intent of this paper 
is to add to these consequences the observational evidence of PL and MC occurrence over the Eastern part of the Eurasian Arctic.

According to [3], polar lows are small, but fairly intense maritime cyclones with the horizontal scale less than $1000 \mathrm{~km}$ and surface winds near or above gale force. The threshold of $\mathrm{V}_{0}$ for maximum wind speeds is used to distinguish PLs between weaker vortices and the more vigorous systems. In [3] $\mathrm{V}_{0}=15 \mathrm{~m} / \mathrm{s}$, but some investigators use other threshold values: $18 \mathrm{~m} / \mathrm{s}$ in [4], $13.9 \mathrm{~m} / \mathrm{s}$ in [5]. Some authors suppose that the presence of cold air outbreaks is the necessary condition to relate a mesocyclone to the class of PLs [6]. Other write about a "spectrum" of polar lows due to a variety of forcing mechanisms, important for the development of these systems [3]. These extreme storms, characterized by strong and rapidly changing winds, are known to enhance vertical mixing processes. This can affect the cold halocline layer, possibly leading to a positive feedback to impact sea-ice formation [7]. Investigating these events is thus not only driven by their destructive power but also by their interconnection with the Arctic climate change and the main climatological cycles [8]. Recent study [9] emphasized the importance of meso-cyclones for the large-scale ocean circulation, as small-scale cyclones can have a significant effect on the northward transport of heat to the northern Europe and North America. A predicted decrease in the Arctic PL number and intensity due to faster positive changes in the air temperature than in the sea surface temperature [10] may then bring cooling to the northern Europe and North America. Yet, so far, reported climatological studies did not prove out model predictions: small positive trends are found both for the PLs over the Nordic and Barents Seas [11], [12] and over the North Pacific seas [13]. In [11], a high correlation is reported between the sea ice extent and the number of identified polar lows. While the stability of the near surface air layer is expected to increase, diminishing the number and intensity of PLs [10], the rapid evolution of the Arctic sea ice extent [14] further opens new regions for the PL formation. This is confirmed by recent findings of new areas of PL formation over the Nordic Seas [12].

Quite a lot of PL studies are dedicated both to the Canadian maritime Arctic and to the Western part of the Eurasian Arctic area. PLs over such seas as the Nordic Seas and the Barents Sea have been investigated in details in many studies since 1960. Objective and subjective PL climatologies are derived both from model and observational data [5], [11], [12], [15]- 
[17] providing extensive data on PL parameter distribution over this sector of the Arctic. Though all the climatological studies differ in their quantitative findings, this is mostly due to apparently different definitions of a polar low which they apply, and various methods used for PL identification. Few studies address PLs over the Chukchi Sea [18]-[20]. The Laptev Sea and the East Siberian Sea have never been mentioned in the scientific literature as possible areas of PL formation. In 2003 Erik Rasmussen and John Turner reviewed in details existing PL studies all over the world and wrote about several isolated cases of reported polar lows over such seas as the Beaufort Sea, the Chukchi Sea and the Kara Sea [3]. According to [3], PLs will be very unlikely found in any new marginal seas around the Arctic Ocean.

The sea ice retreat necessitates revising this conclusion. The general objective of this paper is to report about the common occurrence of PLs over the Eastern part of the Eurasian Arctic and to provide the evidences about the PL cases over such Arctic seas, as the Laptev Sea and the East Siberian Sea. Less intensive mesocyclones are referred to as PLs, basing on 12 $\mathrm{m} / \mathrm{s}$ threshold value of wind speed as opposed to $15 \mathrm{~m} / \mathrm{s}$, which is commonly used. The region of the study is presented in Fig. 1, with the sea ice concentration maps on October 1, in 2002(a) and in 2014(b), illustrating the grown up area of open water in the considered seas for this day.

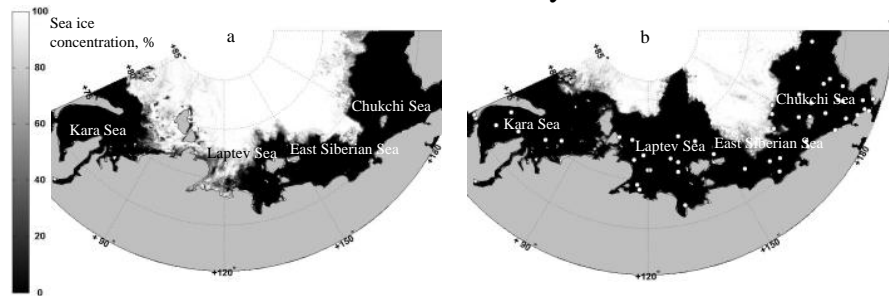

Fig. 1. A map of the Eastern part of the Eurasian Arctic with daily AMSR sea ice concentration (SIC) maps, produced by the Institute of Environmental Physics (IUP) at the University of Bremen [25]: (a) 2002, 1 October, (b) 2014, 1 October. White circles show the approximate positions of the most intensive MCs in their mature phase.

\section{Method AND DATA}

A multi-sensor approach combines data from various sensors taking advantage of their different key strengths and temporal resolution. In this study, PLs are identified using infrared (IR) and visible spectro-radiometer images, and data from passive and active microwave space-borne instruments. Satellite passive microwave observations are used to derive the fields of total atmospheric water vapor content (WVC), cloud liquid water content (CLW) and sea surface wind speed (SWS). As shown in [21], PLs can be identified in WVC fields, even when there are upper clouds to shield PL cloud vortex, inhibiting PL detection in IR and visible images. However IR and visible data can catch much smaller details about the cloud structure than WVC fields based on passive microwave data. Sea surface wind estimates are mandatory in PL studies to evaluate the intensity of a meso-cyclone. In this study we used a threshold of $V_{0}=12 \mathrm{~m} / \mathrm{s}$ to separate PLs from less intensive mesocyclones. Precise SWS data can be problematic to obtain due to relatively low spatial resolution of satellite microwave instruments, especially for PL development near the sea ice boundary. Both satellite scatterometer and radiometer SWS estimates are then used to ensure that not only cloud or water vapor vortexes are detected but real polar low cases are identified with gale force winds. With the advent of many polar orbiting instruments, this synergistic use of both passive and active microwave satellite observations provides means to improve spatiotemporal samplings to investigate PL and MC intensity evolution.

The method of PL identification is thus based on the analysis of multi-sensor data information. Satellite data for the summer and autumn months of 2003 - 2015 years were analyzed on a sample basis for the Kara Sea, the Laptev Sea, the East Siberian Sea and the Chukchi Sea. Aqua and Terra MODIS IR (1 km resolution) and visible ( $250 \mathrm{~m}$ resolution) images were built from the MODIS Level 1B Calibrated Radiances. Mesocyclones were identified by preliminary eye inspection of MODIS images aimed at the detection of cloud systems with a cyclonic curvature and a diameter of less than $1000 \mathrm{~km}$, and the following check of wind speeds retrieved from satellite active and passive microwave measurement data. If the wind speed reached $12 \mathrm{~m} / \mathrm{s}$ in any sector of a mesocyclone, we diagnosed the case of a polar low. Data of the Advanced Microwave Sounding Radiometer - Earth Observing System (AMSR-E) onboard Aqua and the AMSR2 onboard GCOM-W1 satellites were used to derive and analyze the fields of WVC, CLW and SWS. These geophysical parameters were retrieved from AMSR-E and AMSR2 Level 1B swath brightness temperature (BT) data, supplied by the GCOM-W1 Data Providing service. The advanced retrieval algorithms [22], [23] with high accuracies under adverse weather conditions, including both high winds and heavy clouds with large CLW values, were explored. The accuracy of WVC retrievals is critical for PL detection due to low WVC variations over a $\mathrm{PL}$ [21]. If $\mathrm{WVC}$ variations are of the retrieval error order, a PL cannot be discernable in the WVC field.

After a cloud or WVC vortex had been detected, SWS fields were analyzed - both derived from AMSR-E and AMSR2 measurement data and scatterometer products, based on Metop-A, B ASCAT and QuikSCAT SeaWinds data. Maximum winds indicate the intensity of a meso-cyclone and categorizing it as being or not a polar low. At this, the problem of high wind retrieval errors is well known, widely discussed [24] and directly relates to PL studies. Daily AMSR2 sea ice concentration (SIC) maps [25] were used as a source of sea ice edge data.

Additionally to satellite data, surface analysis maps SRRS Analysis and Forecast Charts NOAA along with ERA Interim reanalysis mean sea level pressure (MSLP) and upper air maps [26] were analyzed to deduce the synoptic situations for the detected PLs. The differences between the sea surface temperature (SST) and the air temperature at $2 \mathrm{~m}$ (DT1) and between the SST and the air temperature at the height of 500 $\mathrm{mB}$ pressure (DT2) along with MSLP fields were used to estimate the atmospheric stratification and conditions favorable for the convection vortex rise. ERA Interim MSLP 
fields were also analyzed to search for an associated minimum for considered PL cases.

Objectively Analyzed air-sea Fluxes for the global oceans (OAFlux) from the Woods Hole Oceanographic Institution OAFlux project were also explored to analyze the heat fluxes for the detected PLs.

\section{EXAMPLES OF POLAR LOWS}

One of the PLs over the Laptev Sea is shown in Fig. 2. A clearly identified vortex structure can be detected in both the WVC, retrieved from the Aqua AMSR-E data (Fig. 2(a)), and in the Terra MODIS IR image (Fig. 2(b)). ERA Interim data analysis (baric topography and surface analysis) reveals that this PL of the spiral cloud type formed near the New Siberian Islands in the front of the trough, spreading on October 11, 2007 on the north-west periphery of the deep low over the Bering Sea with the travelling speed of $20 \mathrm{~km} / \mathrm{h}$. This caused the formation of the occlusion-like front and the development of the mesocyclone circulation at its end. This circulation expanded up to $500 \mathrm{mB}$. This PL developed beneath a cold upper vortex under favorable conditions: DT1 $\sim 4-5^{\circ} \mathrm{C}$, DT2 $37^{\circ} \mathrm{C}$. In the end of October 12 the PL crossed the island Kotelny and came over the Laptev Sea. There this PL fell under the influence of the strong cyclone circulation with the center over the Kara Sea. Its travelling speed increased and the maximum winds reached $18 \mathrm{~m} / \mathrm{s}$ during the night of 13 October (Fig. 2(c)). The wind speed development can be traced with high time resolution with QuikSCAT (Fig. 2(d)) and AMSR-E SWS fields. This polar low arose under the total fluxes of heat of about $110-130 \mathrm{~W} / \mathrm{m}^{2}$ and reached the Laptev Sea with significantly lower fluxes values of $80-100 \mathrm{~W} / \mathrm{m}^{2}$. The sea surface temperature of the Laptev Sea was only about $1-2^{\circ} \mathrm{C}$.

The next example refers to the system of meso-cyclones over the East Siberian Sea formed in the central part and rear of the strong occluded cyclone with two centers - over the Chukchi Sea (seen in ERA-Interim MSLP map in Fig. 3(a)) and over the Beaufort Sea on 29 September 2015.
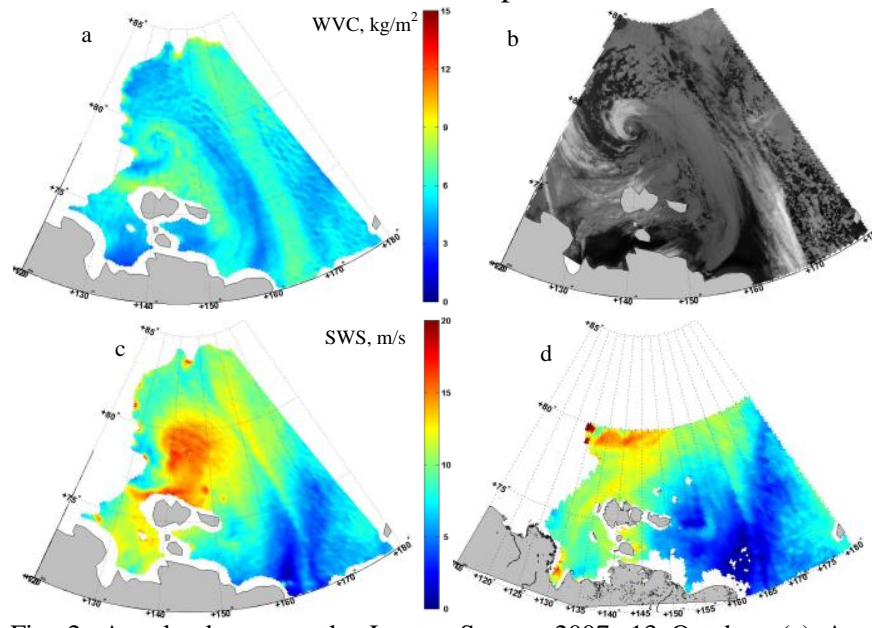

Fig. 2. A polar low over the Laptev Sea on 2007, 13 October: (a) Aqua AMSR-E WVC $\left(\mathrm{kg} / \mathrm{m}^{2}\right)$ at $~ 01: 27$ UTC; (b) Terra MODIS IR image at 02:45 UTC; (c) Aqua AMSR-E SWS (m/s), at 01:27 UTC; (d) QuikSCAT SeaWinds SWS $(\mathrm{m} / \mathrm{s})$ at 09:37 UTC.
MODIS infrared image at 00:15 UTC (Fig. 3(c)) shows the "merry-go-round" system of polar lows [3] $l$ in the central part of the occluded cyclone formed due to convective instability (DT2 $\sim 43^{\circ} \mathrm{C}$ ). Close in time ASCAT wind field shows the maximum winds up to $18 \mathrm{~m} / \mathrm{s}$ in the PLs (Fig. 3(d)). Separate small polar lows are not resolved in the ASCAT SWS field. Favorable conditions to the west off the cyclone center (DT2 $39-40^{\circ} \mathrm{C}$ ) led also to the formation of the chain of three meso-cyclones seen in the MODIS infrared image at 15:10 UTC (Fig. 3(e)). Meso-cyclones 2 and 3 are featured by strato-cumulus and thin cumulus clouds, whereas the vortices 4, formed near the sea ice edge (Fig. 3(b)) in the baroclinic zone of the atmospheric boundary layer have dense, more developed vertically convective clouds. The fast evolution of these meso-cyclones can be investigated with AMSR2 retrieved parameters (Fig. 4(f)) since their development is successfully covered by several consecutive AMSR2 paths. In spite of favorable thermodynamic conditions these mesocyclones did not reach significant intensity: AMSR2 retrieved SWS did not exceed $12 \mathrm{~m} / \mathrm{s}$.

The formation of the mesoscale vortices in the central part of the occluded cyclones and at the fronts of occlusion is the mechanism typical for traditional areas of PL occurrence [3].

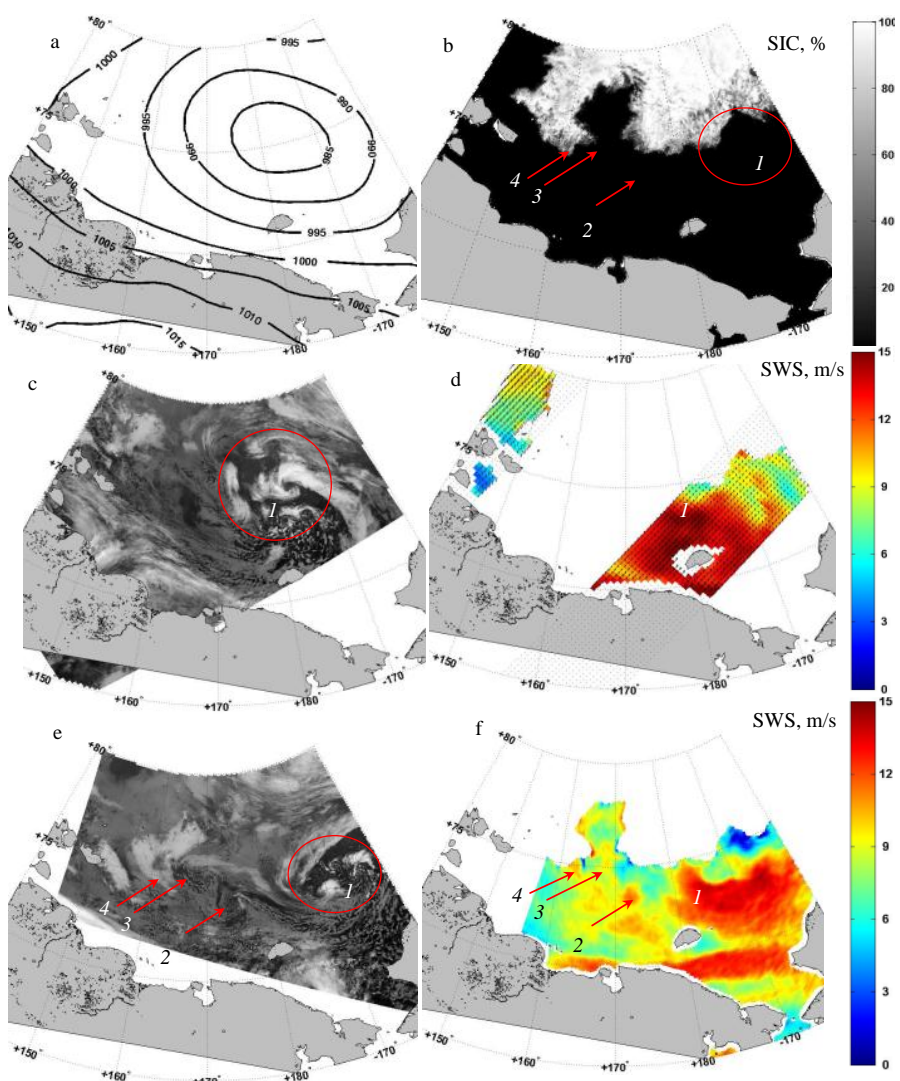

Fig. 3. A system of polar lows over the East Siberian Sea on 2015, 29 September: (a) ERA-Interim MSLP, 00:00 UTC; (b) AMSR2 SIC (\%); (c) Aqua MODIS IR image at $~ 00: 15$ UTC; (d) MetOp-B ASCAT SWS $(\mathrm{m} / \mathrm{s})$ at $\sim$ 00:50 UTC; (e) Aqua MODIS infrared image at 15:10 UTC; (f) GCOMW1 AMSR2 SWS $(\mathrm{m} / \mathrm{s})$ at $~ 15: 10$ UTC. 


\section{RESULTS AND CONCLUSION}

Using multi-sensor approach we analyzed selectively a large archive of satellite data aiming at the case studies of the polar lows over the Eastern part of the Eurasian Arctic. The mesoscale atmospheric activity in this sector of the Arctic is poorly studied, and such a research was initiated by the suggestion that the Arctic sea ice retreat had revealed more open sea water areas susceptible to PL danger. At the very beginning we were not aware if there would be any PLs over the seas which had never been mentioned as possible areas of PL development (the Laptev Sea and the East Siberian Sea). This study reveals the common occurrence of polar MCs and PLs over the marginal seas of the Eastern part of the Eurasian Arctic covering the Kara Sea, the Laptev Sea, the East Siberian Sea and the Chukchi Sea.

Detailed case studies can efficiently build on the combined use of multi-sensor satellite observations, surface analysis and upper air maps and ERA Interim model to more particularly scrutinize the mesoscale activity over the considered area. Whereas PLs over the Kara Sea and the Chukchi Sea are known to occur, their general existence over the Laptev Sea and the East Siberian Sea is newly detected and needs to be further investigated.

Expecting less favorable conditions for PL development than those over the traditional areas of PL development (e.g. the Nordic Seas and the Barents Sea), we lowered the threshold value for the wind speed to $12 \mathrm{~m} / \mathrm{s}$ to diagnose a PL case after a cloud vortex had been identified in a MODIS IR or visible image. In the most PL studies the value of $15 \mathrm{~m} / \mathrm{s}$ is used to distinguish between PLs and less intensive mesoscale vortices. The author expedition and prognostic work experience evidences that the winds of $12 \mathrm{~m} / \mathrm{s}$ already pose a threat to small vessels. Besides, satellite based wind speed estimation over the Arctic cold open seas suffer from the underestimation of the real winds [27]. $12 \mathrm{~m} / \mathrm{s}$, estimated from satellite active or passive microwave measurement data can in reality be as high as $15 \mathrm{~m} / \mathrm{s}$ and even larger.

Though in [21] it was shown that polar lows could be definitely identified in integrated water vapor content fields, we established that the considered PLs over the Eastern part of the Eurasian Arctic, detected in IR and visible images, did not show well in the AMSR2 WVC fields. This might be associated with extremely low WVC values in the regions and low MC intensities comparatively to traditional areas of $\mathrm{PL}$ development. Also, PLs and MCs of smaller sizes are distinctive for the considered geographical area. Still WVC and CLW numerical data, retrieved from AMSR2 measurement data, contribute to better analysis of PL evolution. The combined usage of both satellite passive and active microwave sea surface wind estimates has demonstrated the potential of the highest temporal resolution in the investigation of PL intensity. At the same time there are inherent complications for SWS estimation in PLs over the considered seas. Many identified PLs arose over the sea ice edge and evolved close to the sea ice, where satellite SWS retrievals are impossible. The following research progress is associated with the development of the AMSR2 retrieval algorithms, valid over the sea ice edge.

Currently we analyzed more than $150 \mathrm{MC}$ cases for August, September, October and November months. Not all the years of 2003 - 2015 were investigated with equal elaboration. Most complete archive of data was created for 2007 and 2014 years. Since not all the data were processed, we make no conclusion about the growing activity of PLs over the considered area. We can only assume that when these areas were mostly covered by the sea ice, no PLs could develop. This is indirectly vouched by the lack of references to these events. Our preliminary analysis either does not support the idea of significant correlation between the open water area and the PL number or intensity.

This initial study of the PLs over the Eastern part of the Eurasian Arctic shows that about $40 \mathrm{MCs}$ a year can develop over the Kara Sea, the Laptev Sea, the East Siberian and the Chukchi Sea in August - November months, when the mesoscale activity is most probable in this Arctic area. To illustrate this statement, the approximate positions of the most intensive MCs over the Eastern part of the Eurasian Arctic in 2014 in their mature phase are shown in Fig. 1(b) as white circles.

A distinctive feature of the synoptic processes over the Eastern part of the Eurasian Arctic is their particular isolation due to the area remoteness from the main cyclogenesis zones of the Northern Hemisphere. Cyclones, formed directly over the Arctic waters, are less intense and smaller than the cyclones of middle latitudes. Some of them are a continuation of the synoptic processes, developing over the Far Eastern seas [20]. These cyclones occlude and dissipate over the Eastern part of the Eurasian Arctic. The cold air outbreaks in this region are not so intense and sustained, as over the traditional areas of PL spreading. However the spacious sea ice fields are the source of persistent cold advection and formation of shallow baroclinic zones along their edges. The large-scale atmospheric processes influence on the mesoscale cyclonic activity over the considered region. In this region meso-cyclones occur mainly in the shallow baroclinic zones near the sea ice edge at continuous cold air advection from the sea ice-covered regions of the Arctic Ocean and in the central part of the old occluded cyclones under the cold high-level depressions. In addition, under certain wind direction orographic PLs may form over the bays of the Arctic and Bering Sea coast [20].

Though more studies are needed to make statistical conclusions, our preliminary analysis shows that in this sector of the Arctic MCs and PLs with spiral form clouds and the size, not exceeding 200-300 km, prevail. $90 \%$ of the considered MCs and PLs have spiral form clouds, the other $10 \%$ are comma cloud cyclones. The heat fluxes analysis showed that all detected MCs developed under significantly lower values of heat fluxes than commonly expected. It is found also that in the current climate PLs emerge over the Eastern part of the Eurasian Arctic mostly in the fall.

Many recent PL studies estimate the ability of reanalysis data to reproduce mesocyclones [28], [29]. While the mean sea level pressure fields are well captured by various ERA 
reanalysis data sets to agree well in synoptic-scale cyclone timing and location, mesoscale lows are much more challenging [2]. We found that only about $10 \%$ of the considered PLs over the Eastern part of the Eurasian Arctic showed an associated minimum in mean sea level pressure in ERA Interim reanalysis fields. This is much lower than 55\% of satellite observed PLs both in [28] and 45\% of satellite observed PLs in [29]. This might be associated both with less intensive class of considered mesocyclones and with other factors specific for the PLs over the study area, e.g. lower sea surface temperatures, closer sea ice etc. The use of operational forecast models, e.g. ECMWF Integrated Forecast System, with higher horizontal resolution should be more appropriate for PL detection. But to quantify this statement further investigation is needed.

The seasonally expected sea ice character with a companion intensification of the meridional atmospheric circulation will probably further lead to more frequent passages of deep Pacific Ocean cyclones over the Arctic coast [30]. Again, this will intensify cyclonic and mesocyclonic activity in the Eastern part of the Eurasian Arctic. These processes need to be further studied as highly important for both the Arctic shipping, marine and coastal industries, and for understanding the Arctic climate and its current and future changes.

\section{REFERENCES}

[1] D. J. Cavalieri and C. L. Parkinson, "Arctic sea ice variability and trends, 1979-2010," Cryosphere, vol. 6, no. 4, pp. 881-889, 2012.

[2] T. Vihma, "Effects of Arctic sea ice decline on weather and climate: A review," Surv. Geophys., vol. 35, no. 5, pp. 1175-1214, 2014

[3] E. A. Rasmussen and J. Turner, Polar lows: mesoscale weather systems in the polar regions. Cambridge: Cambridge University Press, 2003.

[4] R. W. Fett, R. E. Englebretson, and D. C. Perryman, "Forecasters Handbook for the Bering Sea, Aleutian Islands, and Gulf of Alaska," DTIC Document, 1993.

[5] M. Zahn and H. von Storch, "A long-term climatology of North Atlantic polar lows," Geophys. Res. Lett., vol. 35, no. L22702, 2008.

[6] E. W. Kolstad, "A global climatology of favourable conditions for polar lows," Q. J. R. Meteorol. Soc., vol. 137, no. 660, pp. 1749$1761,2011$.

[7] V Ivanov, V. A. Alexeev, T. A. Alexeeva, N. V. Koldunov, I. A. Repina, and A. V. Smirnov, "Is the Arctic sea ice becoming seasonal?," Earth Observation from Space, no. 4, pp. 50-65, 2013. (in Russian)

[8] P.-E. Mallet, C. Claud, C. Cassou, G. Noer, and K. Kodera, "Polar lows over the Nordic and Labrador Seas: Synoptic circulation patterns and associations with North Atlantic-Europe wintertime weather regimes," J. Geophys. Res. Atmospheres, vol. 118, no. 6, pp. 2455-2472, Mar. 2013

[9] A. Condron and I. A. Renfrew, "The impact of polar mesoscale storms on northeast Atlantic Ocean circulation," Nat. Geosci., vol. 6, no. 1, pp. 34-37, 2012.

[10] M. Zahn and H. von Storch, "Decreased frequency of North Atlantic polar lows associated with future climate warming," Nature, vol. 467, no. 7313, pp. 309-312, 2010.

[11] J. E. Smirnova, P. A. Golubkin, L. P. Bobylev, E. V. Zabolotskikh, and B. Chapron, "Polar low climatology over the Nordic and Barents seas based on satellite passive microwave data," Geophys. Res. Lett.,
doi:10.1002/2015GL063865, 2015.

[12] M. Rojo, C. Claud, P.-E. Mallet, G. Noer, A. M. Carleton, and M. Vicomte, "Polar low tracks over the Nordic Seas: a 14-winter climatic analysis," Tellus A, vol. 67, no. 0, Apr. 2015.

[13] F. Chen and H. von Storch, "Trends and Variability of North Pacific Polar Lows," Adv. Meteorol., vol. 2013, p. e170387, Jun. 2013.

[14] J. C. Comiso, "Large Decadal Decline of the Arctic Multiyear Ice Cover," J. Clim., vol. 25, no. 4, pp. 1176-1193, Feb. 2012.

[15] A. M. Blechschmidt, "A 2-year climatology of polar low events over the Nordic Seas from satellite remote sensing," Geophys. Res. Lett., vol. 35 , no. $9,2008$.

[16] T. J. Bracegirdle and S. L. Gray, "An objective climatology of the dynamical forcing of polar lows in the Nordic seas," Int. J. Climatol., vol. 28, no. 14, pp. 1903-1919, 2008.

[17] G. Noer, Ø. Saetra, T. Lien, and Y. Gusdal, "A climatological study of polar lows in the Nordic Seas," Q. J. R. Meteorol. Soc., vol. 137, no. 660 , pp. $1762-1772$, Oct. 2011

[18] J. Inoue, M. E. Hori, Y. Tachibana, and T. Kikuchi, "A polar low embedded in a blocking high over the Pacific Arctic," Geophys. Res. Lett., vol. 37, no. 14, p. L14808, Jul. 2010.

[19] P. D. Moreira, "Mesoscale Modeling Study of a Polar Low in the Chukchi and Beaufort Seas," University of Alaska Fairbanks, 2011.

[20] P. I. Zimich, Atmospheric processes and weather of Eastern Arctic. Vladivostok: Dalnauka, 1998, 236 P. (in Russian).

[21] L. P. Bobylev, E. V. Zabolotskikh, L. M. Mitnik, and M. L. Mitnik, "Arctic Polar Low Detection and Monitoring Using Atmospheric Water Vapor Retrievals from Satellite Passive Microwave Data," IEEE Trans. Geosci. Remote Sens., vol. 49, no. 9, pp. 3302-3310, 2011

[22] L. P. Bobylev, E. V. Zabolotskikh, L. M. Mitnik, and M. L. Mitnik, "Atmospheric water vapor and cloud liquid water retrieval over the Arctic Ocean using satellite passive microwave sensing," IEEE Trans. Geosci. Remote Sens., vol. 48, no. 1, pp. 283-294, 2010.

[23] E. V. Zabolotskikh, L. M. Mitnik, and B. Chapron, "New approach for severe marine weather study using satellite passive microwave sensing," Geophys. Res. Lett., vol. 40, no. 13, pp. 3347-3350, 2013.

[24] E. Zabolotskikh, L. Mitnik, and B. Chapron, "GCOM-W1 AMSR2 and MetOp-A ASCAT wind speeds for the extratropical cyclones over the North Atlantic," Remote Sens. Environ., vol. 147, pp. 8998, May 2014.

[25] G. Spreen, L. Kaleschke, and G. Heygster, "Sea ice remote sensing using AMSR-E 89-GHz channels," J. Geophys. Res. Oceans 19782012, vol. 113, no. C2, 2008.

[26] D. P. Dee, S. M. Uppala, A. J. Simmons, P. Berrisford, P. Poli, S. Kobayashi, U. Andrae, M. A. Balmaseda, G. Balsamo, P. Bauer, and others, "The ERA-Interim reanalysis: Configuration and performance of the data assimilation system," $Q$. J. R. Meteorol. Soc., vol. 137, no. 656, pp. 553-597, 2011.

[27] S. A. Grodsky, V. N. Kudryavtsev, A. Bentamy, J. A. Carton, and B. Chapron, "Does direct impact of SST on short wind waves matter for scatterometry?," Geophys. Res. Lett., vol. 39, no. 12, 2012.

[28] G. Zappa, L. Shaffrey, and K. Hodges, "Can Polar Lows be Objectively Identified and Tracked in the ECMWF Operational Analysis and the ERA-Interim Reanalysis?," Mon. Weather Rev., vol. 142 , no. 8, pp. 2596-2608, Apr. 2014

[29] T. Laffineur, C. Claud, J.-P. Chaboureau, and G. Noer, "Polar Lows over the Nordic Seas: Improved Representation in ERA-Interim Compared to ERA-40 and the Impact on Downscaled Simulations," Mon. Weather Rev., vol. 142, no. 6, pp. 2271-2289, Jan. 2014.

[30] J. Bader, M. D. Mesquita, K. I. Hodges, N. Keenlyside, S. Østerhus, and M. Miles, "A review on Northern Hemisphere sea-ice, storminess and the North Atlantic Oscillation: Observations and projected changes," Atmospheric Res., vol. 101, no. 4, pp. 809-834, 2011. 